\title{
Two New Stenosis Detection Methods of Coronary Angiograms
}

\author{
Yaofang Liu • Xinyue Zhang • Wenlong \\ Wan · Shaoyu Liu · Yingdi Liu - Hu Liu • \\ Xueying Zeng • Qing Zhang
}

Received: date / Accepted: date

\begin{abstract}
Purpose Coronary angiography is the "gold standard" for diagnosing coronary artery disease (CAD). At present, the methods for detecting and evaluating coronary artery stenosis cannot satisfy the clinical needs, e.g., there is no prior study of detecting stenoses in prespecified vessel segments, which is necessary in clinical practice.

Methods Two vascular stenosis detection methods are proposed to assist the diagnosis. The first one is an automatic method, which can automatically extract the entire coronary artery tree and mark all the possible stenoses. The second one is an interactive method. With this method, the user can choose any vessel segment to do further analysis of its stenoses.

Results Experiments show that the proposed methods are robust for angiograms with various vessel structures. The precision, sensitivity, and $F_{1}$ score of the automatic stenosis detection method are $0.821,0.757$, and 0.788 , respectively. Further investigation proves that the interactive method can provide a more precise outcome of stenosis detection, and our quantitative analysis is closer to reality.

Conclusion The proposed automatic method and interactive method are effective and can complement each other in clinical practice. The first method can be used for preliminary screening, and the second method can be used for further quantitative analysis. We believe the proposed solution is more suitable for the clinical diagnosis of CAD.
\end{abstract}

Keywords Coronary angiograms · Coronary artery diseases · Automatic stenosis detection · Interactive stenosis detection

\section{Introduction}

Coronary angiography (CA) is the "gold standard" in clinical diagnosis of coronary artery disease (CAD) [1, which is the leading cause of death worldwide 2, affecting over 120 million people 3]. At present, the methods for identifying coronary artery lesions, i.e., stenoses, and evaluating the stenotic degree are mostly based on the subjective estimation of doctors. It results in a large number of repetitive work that not only reduces the work efficiency but also causes significant subjective errors, which can lead to fault judgments 4. Aimed to solve these problems, people have proposed various computer-aided diagnosis methods.

Qing Zhang

Department of Cardiology, Qilu Hospital (Qingdao), Cheeloo College of Medicine, Shandong University, Qingdao, Shandong, China.

E-mail: qingzhang2019@foxmail.com

Yaofang Liu · Xinyue Zhang · Yingdi Liu · Xueying Zeng

School of Mathematical Sciences, Ocean University of China, Qingdao, Shandong, China

Wenlong Wan · Shaoyu Liu

School of Computer Science and Technology, Ocean University of China, Qingdao, Shandong, China

$\mathrm{Hu} \mathrm{Liu}$

School of Material Science and Engineering, Ocean University of China, Qingdao, Shandong, China 
Most researchers focused on approaches for the automatic assessment of CAD. A generic framework of these approaches is as follows: (1) coronary artery tree extraction, (2) diameter calculation, and (3) analysis of the stenotic segment. The key stage that determines the speed and accuracy of such algorithms is based on coronary artery tree extraction. The related techniques can be broadly divided into three categories, including the tracking-based methods [5] 7], e.g., Xiao et al. [8] introduced AGVT to extract the vessel centerlines by tracking from the selected seed points, using the local gray distribution and geometrical information, e.g., Frangi et al. 9] proposed a common vesselness enhancement technique, where the multiscale second-order local structure of an image (Hessian) is examined, and deep learning methods [10 13], e.g., NasrEsfahani et al. [14] designed a framework consisting of two convolutional neural networks $(\mathrm{CNN})$, where the first CNN uses local and global image patches to establish an initial segmentation probability map, and the input of the second CNN includes the output of the first $\mathrm{CNN}$ as well as edge information from canny edge detector. The last kind is acknowledged promising as it is a powerful tool in computer vision [15] and has achieved higher efficiency and accuracy in similar tasks like vessel segmentation of retinal image [16 22. These approaches are very demanding on datasets because their performance depends largely on the accuracy of the dataset. However, it is time-consuming and burdensome to generate ground truth data for the angiography images, and there is no public dataset for this task 11.

Another popular framework for automatically assessing CAD is using machine/deep learning methods to localize the stenoses directly. In prior work 23, stenosis bounding boxes were annotated in images as stenosis localization labels. It introduced an endto-end CNN-based workflow, where CNN and recurrent neural network models were employed for coronary artery view classification, candidate frame selection, and imagelevel stenosis classification. Ovalle-Magallanes et al. 24 proposed a network-cut and fine-tuning hybrid method for stenosis detection in coronary angiograms employing a pre-trained CNN via transfer learning (VGG16, ResNet50, and Inception-v3). Aimed at confirming the feasibility of real-time coronary artery stenosis detection using deep learning methods, Danilov et al. 15] trained eight detectors based on different neural network architectures (MobileNet, ResNet-50, ResNet-101, Inception ResNet, NASNet) to detect the location of stenoses using angiography imaging series and assessed their performance. Nevertheless, these methods have the identical drawback: they can't give a quantitative result of the stenoses, e.g., the stenotic degree, which means they cannot meet the quantitative analysis needs for clinical diagnosising CAD.

Apart from the problems mentioned above, another considerable defect is that these existing methods only did global stenosis detection in the angiographic image. However, medical staff needs to test a particular vascular segment for more detailed and accurate detection and quantitative analysis in practical application. In fact, to the best of our knowledge, there is no prior study of detecting stenoses in prespecified vessel segments.

Considering these issues, we introduce a novel solution that integrates automatic and interactive stenosis detection methods in this paper. Specifically, we first propose an automatic stenosis detection method to detect stenoses of the whole coronary artery tree. As for extracting the artery tree, we introduce an improved adaptive geometrical tracking algorithm (IAGVT), which combines the advantages of tracking-based methods and filtering-based methods. On top of this global detection method, we further designed an interactive stenosis detection method that allows medical staff to choose any vessel segment to analyze stenoses more precisely. It uses an energy function to track the centerline of the prespecified vessel segment. The first automatic method can be used for preliminary screening, and the interactive method can be used for further quantitative analysis. We argue that our solution is more suitable for the clinical diagnosis of CAD.

\section{Methods}

This section will propose two stenosis detection methods: an automatic one and an interactive one. Firstly, we introduce the fundamental algorithms of the two methods. 
Preparation

Overall, to detect stenoses, we first segment the vessels. Then, we apply our methods of diameter measurement and stenotic degree evaluation to every part of the vessels. Finally, we identify the stenoses by comparing the stenotic degrees with a given standard. In this section, we propose the fundamental methods to do so.

Image preprocessing. Following Xiao et al. [8, we use a multiscale image enhancement method [9] to highlight the vascular structure. However, it brings huge difficulty to accurately segment vessels that the complex and variable shape of coronary artery structure, noise caused by various factors, etc. Therefore, we propose an image preprocessing scheme including Rudin-Osher-Fatemi (ROF) [25], unsharp masking (UM) 26], and contrast limited adaptive histogram equalization (CLAHE) 27. Specifically, ROF is used for image denoising as it has proved to be one of the most successful tools to do such work 28]. Besides, we introduce UM, a classical tool for sharpness enhancement [29], to further highlight the blood vessels. Moreover, we use CLAHE to enhance the color retinal image. This enhancement method is widely used in ophthalmology, such as the automatic detection of micro aneurisms and retinal blood vessel segmentation 30. Extract the vessel centerline. As prior efforts [8] show, the adaptive geometrical tracking algorithm (AGVT) is effective in this task. Based on it, we introduce an improved adaptive geometrical tracking algorithm (IAGVT).

To start the tracking, we need to get a seed point. Following AGVT, we first detect ridge points of the vascular structure, which are the gray maximum points perpendicular to the direction of the vessels. The gradient of the local maximum point in the image is zero, and its Hessian matrix is negative. Thus, if the point $(\varepsilon, \eta)(x<\varepsilon<x+1, y<$ $\eta<y+1)$ satisfies the following conditions:

$$
\begin{aligned}
& \nabla(x, y) \nabla(x+1, y+1)<0 \text { or } \\
& \nabla(x+1, y) \nabla(x, y+1)<0, \\
& \lambda_{i}(x+m, y+n)<0,(i=1,2, m=0,1, n=0,1),
\end{aligned}
$$

where $\nabla(x, y)$ is the gray gradient of point $(x, y)$ and $\lambda_{i}(x, y)$ are the eigenvalues of the Hessian matrix of point $(x, y)$, then $(\varepsilon, \eta)$ can be considered as a local maximum point. The pixel $(x, y)$, as its approximate solution, is defined as a ridge point. After that, a seed point $P_{0}$ can be randomly chosen from ridge points.

Next, we track by identifying the tracking direction and finding the next tracking point. The initial tracking direction can be obtained from the gray information near the seed point. Concretely, take the seed point as the center and search for the gray maximum point $P^{+}$on the circle with radius $d . P^{+}$is the first point of forward tracking, the forward initial tracking direction $u^{+}$and angle $\theta^{+}$can be expressed as:

$$
u\left(P^{+}\right)=\frac{P^{+}-P}{\left\|P^{+}-P\right\|}=\left(\cos \theta^{+}, \sin \theta^{+}\right)
$$

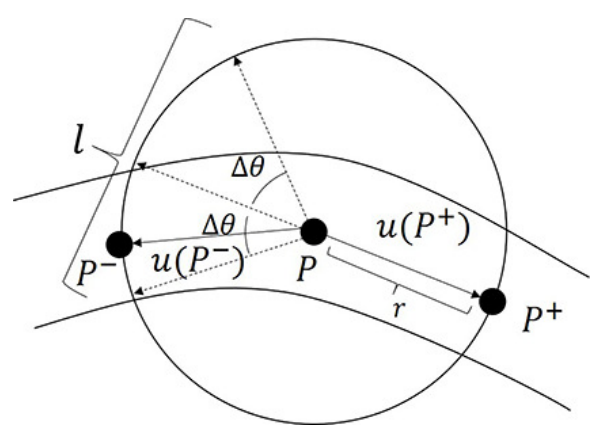

Fig. 1 Initial direction detection

Then, we search for the local maximum point $P^{-}$on $\operatorname{arc} l\left(2 \pi-\theta^{+}-\Delta \theta, 2 \pi-\theta^{+}+\Delta \theta\right)$ centered on the opposite direction $\left(2 \pi-\theta^{+}\right)$of the forward tracking angle $\theta^{+}$. The 
backward direction of the initial tracking $u^{-}$can be calculated as:

$$
u\left(P^{-}\right)=\frac{P^{-}-P}{\left\|P^{-}-P\right\|}
$$

The search process is shown in Fig. 1 .

After that, we keep track from the last point to the next point. Any tracking direction is determined by:

$$
u_{k}=\frac{P_{k}-P_{k-1}}{\left\|P_{k}-P_{k-1}\right\|}
$$

Then, we search for the local maximum point $P_{k+1}$ on $\operatorname{arc} l_{k}\left(\theta_{k}-\Delta \theta, \theta_{k}+\Delta \theta\right)$. To prevent the over-tracking beyond the vessel area in AGVT, we condition $P_{k+1}$ as follows:

$$
\left\{\begin{array}{l}
I\left(P_{k+1}\right)>I_{0} \\
N_{P}\left(P_{k+1}\right)<\tau_{P},
\end{array}\right.
$$

where $I\left(P_{k+1}\right)$ is the gray value of $P_{k+1}, I_{0}, \tau_{P}$ are thresholds, and $N_{P}\left(P_{k+1}\right)$ is the number of tracking points around $P_{k+1}$. The first condition in Eq. 5 is to prevent the over-tracking beyond the vessel area, and the second condition can avoid repeatedly tracking the vessel and being trapped in an endless local loop.

If only rely on gray information to orient, a few tracking points may devi ate from the vascular center as the gray distribution is not uniform in some parts. Different from the centerline adjustment method in 8 , which still relies on the local gray distribution, we incorporate it with the $\mathrm{C}-\mathrm{V}$ model 31 . The $\mathrm{C}-\mathrm{V}$ model is the most popular regionbased active contour model [32, and it identifies vessel and background regions using global region statistical information.

After obtaining the vessel contour with the $\mathrm{C}-\mathrm{V}$ model, the specific adjustment steps are: get the normal line of the vessel through the vertical direction of the current tracking direction, find the intersection points $G_{1}, G_{2}$ of the normal line and the vessel contour, then tracking point $P_{k}$ can be adjusted to:

$$
P_{k}^{\prime}=\frac{G_{1}+G_{2}}{2}
$$

meanwhile, change the tracking direction $u_{k}$ to:

$$
u_{k}^{\prime}=\frac{P_{k}^{\prime}-P_{k-1}}{\left\|P_{k}^{\prime}-P_{k-1}\right\|} .
$$

The adjustment process is illustrated in Fig. 2

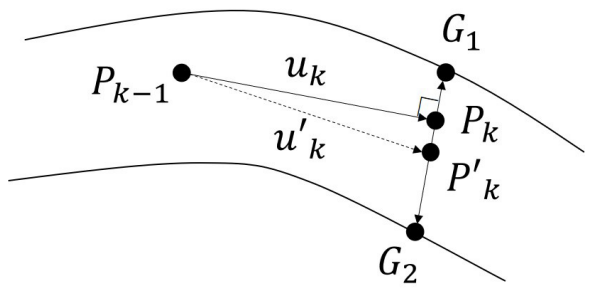

Fig. 2 Centerline adjustment

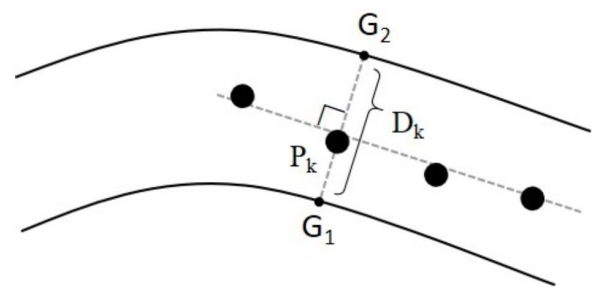

Fig. 3 Vascular diameter measurement 
Diameter measurement and stenotic degree evaluation. We have known how to extract the vascular skeleton and contour. Based on this, we can detect stenoses.

In the previous section, we adjusted the tracking point to the centerline. The diameter of the target vessel tracking point can be measured according to the adjusted vascular skeleton information and the vascular contour extracted. The algorithm flow is as follows

(1) Give any point $P_{k}$.

(2) Calculate distances from each tracking point $P_{i}(i=1,2, \ldots, n)$ on the vascular centerline to $P_{k}: d_{1}, d_{2}, \ldots, d_{n}$.

(3) Sort these distances and select four points with the smallest distances (if $n<4$, select $n$ points).

(4) Fit a line $l_{1}$ that passes through the nearest four $/ n$ points using the least square method.

(5) Obtain the normal line $l_{2}$ of $l_{1}$ at $P_{k}$.

(6) Get the intersection points ( $l_{2}$ with the vessel contour) $I_{1}$ and $I_{2}$.

(7) Calculate the distance $D_{k}$ between $I_{1}$ and $I_{2} . D_{k}$ is the diameter at $P_{k}$.

The measurement schematic diagram is shown in Fig. 3 .

The stenotic degree of the point $P_{i}$ is evaluated as:

$$
S_{i}=\frac{D_{i}}{\bar{D}}
$$

where $\bar{D}$ is the average diameter of the corresponding vessel segment.

To assess the stenosis, we set a discriminant function:

$$
\delta\left(P_{i}\right)=\left\{\begin{array}{ll}
1, & \text { if } S_{i}<\tau_{3}, \\
0, & \text { otherwise }
\end{array},\right.
$$

where $\tau_{3}$ is a threshold. When $\delta\left(P_{i}\right)$ equals zero, the point $P_{i}$ is viewed as stenosis. Following Neubauer et al. [33], $P_{i}$ is identified as stenosis when any substantial and visually evident $(>20 \%)$ reduction in vessel diameter. Thus, we set 0.8 as the default value for $\tau_{3}$ in our later experiments.

Automatic stenosis detection

As mentioned above, firstly, a seed point is randomly chosen from the ridge points. Then, we can get the tracking point sequence of the blood vessels using IAGVT. However, our tracking method can't track the whole vessel structure yet because of the bifurcations. So, we need to add a bifurcation detection process.

This process includes two main steps: first, we obtain one branch point (tracking point) $P_{k}$ by the tracking method. Then, we need to find the other branch point. Different from Xiao et al. [8, which just find another $P_{k}$ using the same conditions, we search in the fan ring area between angle $\left(\theta_{k}-\Delta \theta^{\prime}, \theta_{k}+\Delta \theta^{\prime}\right)$ and radius $\left(r_{1}, r_{2}\right)$ to find a ridge point that satisfies the following conditions:

$$
\left\{\begin{array}{l}
\left|\theta_{b}-\theta_{k}\right|>\tau_{1} \\
\left|\theta_{b}-\theta_{k-1}\right|>\tau_{2} \\
\left\|P_{b}-P_{k}\right\|>d \\
N_{B}\left(P_{b}\right)<\tau_{B},
\end{array}\right.
$$

where $P_{b}$ and $u\left(P_{b}\right)$ represent the detected ridge point of the new branch (the branch point) and direction of it, respectively, $N_{B}\left(P_{b}\right)$ is the number of bifurcations around the branch point $P_{b}$. The first three conditions in Eq. 10 mean that the two branches should differ in several aspects. The last condition indicates that $N_{B}\left(P_{b}\right)$ should be smaller than threshold $\tau_{B}$ to avoid duplication with existing tracking. The bifurcation detection process is shown in Fig. 4 .

Now, we get the tracking point sequence of the whole vessel structure. Due to the diameters of blood vessels varying wildly, we need to segment the vessels before detecting stenoses. 


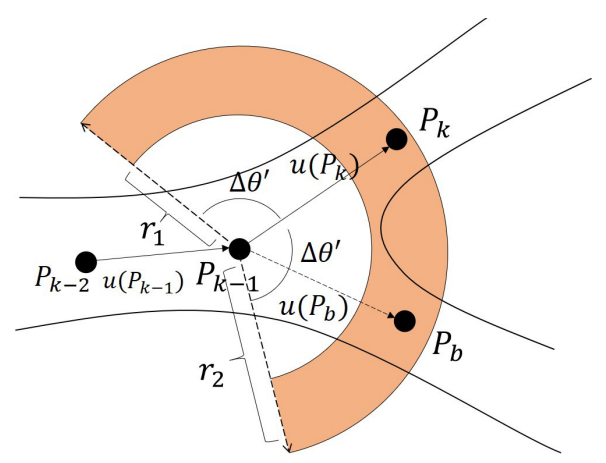

Fig. 4 Vascular bifurcation detection

In the tracking process, the tracking points on each section are continuously tracked from start to end in a geometry sense, so the tracking point sequence of a particular segment is continuous in the whole sequence. Using this feature, we propose a concise and accurate vessel segmenting method. Specifically, we obtain vessel segments by extracting point sequences between every two adjacent cutoff points, including bifurcation points and termination points of the tracking point sequence. A typical tracking process is shown in Fig. 5

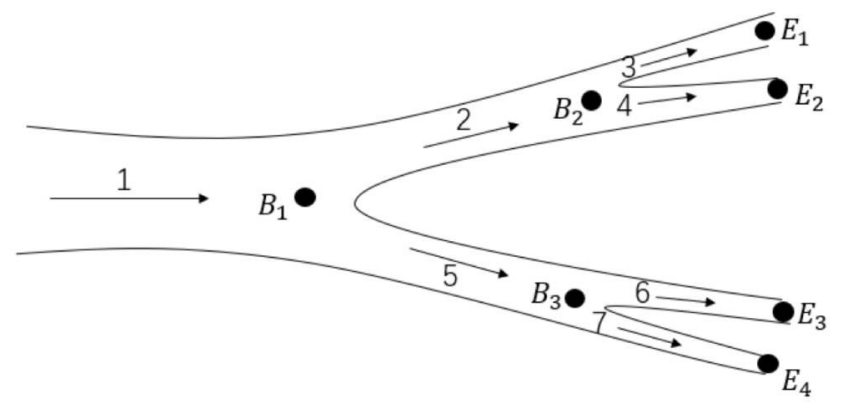

Fig. 5 Tracking process. $B$ represents the bifurcation point, $E$ represents the termination point of piecewise tracking, the arrow indicates the tracking direction, and the number indicates the order of tracking.

Once the segments are obtained, we can detect the stenoses by using the method of diameter measurement and stenotic degree evaluation on each vessel segment.

Interactive stenosis detection

In practical applications, doctors may need to analyze a particular vessel segment of interest. Thus, we propose an interactive detection algorithm, which enables the user to detect and do quantitative analysis of stenoses on a particular segment by only interactively specifying a start point $P_{\text {start }}$ and an endpoint $P_{\text {end }}$. Precisely, we first extract the vessel segment between $P_{\text {start }}$ and $P_{\text {end }}$ based on a proposed energy function. Then, we identify stenoses using our diameter measurement algorithm and Eq.(8) (9) mentioned above.

In extracting the segment, the complicated topology of the vessels is the most challenging problem, especially the bifurcation structure, which can easily make the tracking point deviate from the correct route, i.e., it tracks to the other branch. To solve this problem, rather than using IAGVT directly, we introduce a potential energy function:

$$
E(P)=I(P)+\frac{\lambda}{\sqrt{d\left(P, P_{\text {end }}\right)}},
$$

where $\lambda$ is a coefficient. At every tracking step, we select the point with the largest value of the potential energy function on the search arc as the next tracking point so that 


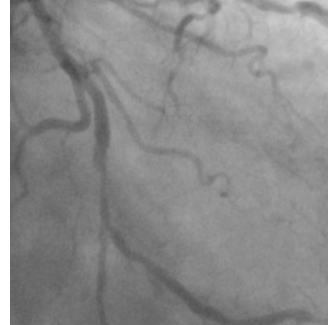

(a1)

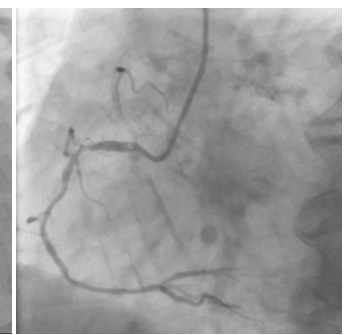

(a2)

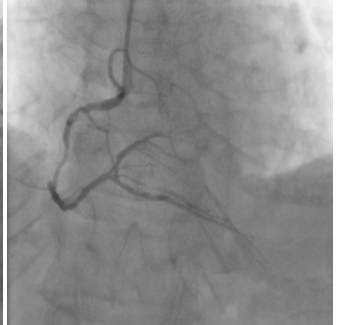

(a3)

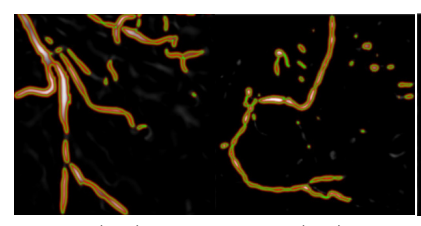

(b1)

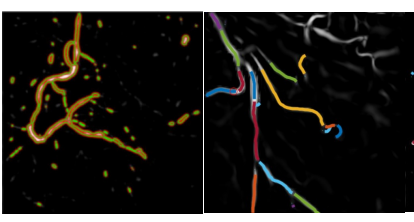

(c1)

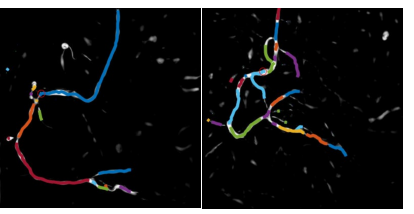

(c2)

(c3)

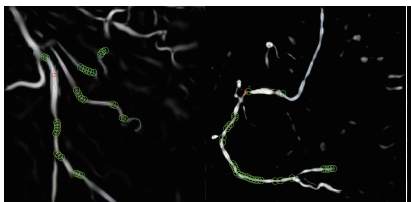

(d1)

(d2)

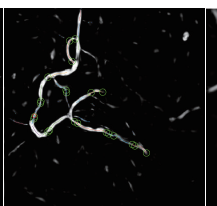

(d3)

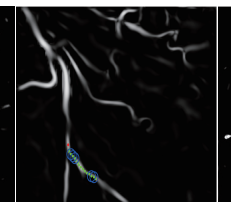

(e1)

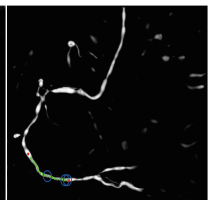

(e2)

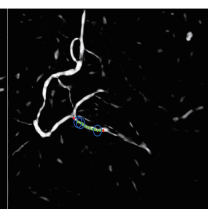

(e3)

Fig. 6 Overall experimental results of our proposed solution. (a) Original images. (b) Vessel contour extraction after image preprocessing. (c) Vessel segmenting. (d) Automatic stenosis detection. (e) Interactive stenosis detection

we can get the correct tracking route. The first item makes the next tracking point not deviate from the vessel. The second item can make the tracking point avoid deviating at the bifurcation and gradually approaching $P_{\text {end }}$.

Using the above method, we significantly improved the effect of interactive detection. The specifical algorithm of interactive stenosis detection is as follows

(1) Specify $P_{\text {start }}$ and $P_{\text {end }}$ interactively.

(2) Obtain the adjacent ridge points. The start point $P_{\text {start }}^{\prime}$ and the end point $P_{\text {end }}^{\prime}$ can be formulated as:

$$
\left\{\begin{array}{l}
P_{\text {start }}^{\prime}=\underset{P \in \mathcal{R}}{\arg \min d}\left(P, P_{\text {start }}\right) \\
P_{\text {end }}^{\prime}=\underset{P \in \mathcal{R}}{\arg \min d}\left(P, P_{\text {end }}\right)
\end{array},\right.
$$

where $\mathcal{R}$ represents the set of the ridge points.

(3) Track. Take $P_{\text {start }}^{\prime}$ as the seed point. The point $P_{j}$ with the largest value of the potential energy function Eq. 111 on the search arc is selected as the next tracking point. Then, we determine whether the distance between the tracking point and $d\left(P_{j}, P_{\text {end }}^{\prime}\right)$ is less than a given threshold $\tau_{d}$. If so, stop tracking. Otherwise, continue.

(4) Select the correct route. Considering there may be a ring structure, we perform step(3) in the forward and backward directions to get two routes and select the one with fewer tracking points as the correct route.

(5) Detect stenoses. We can identify stenoses at each tracking point using our algorithm of diameter measurement and Eq.(8)(9) mentioned above.

\section{Results and discussion}

Dataset and settings

The proposed methods are evaluated on a coronary angiograms dataset consisting of 53 grayscale images sized at $400 \times 400$ pixels with available ground truth for stenosis detection by an expert. The images are selected from CDs in DICOM format of 30 patient cases provided by Qilu Hospital (Qingdao). 


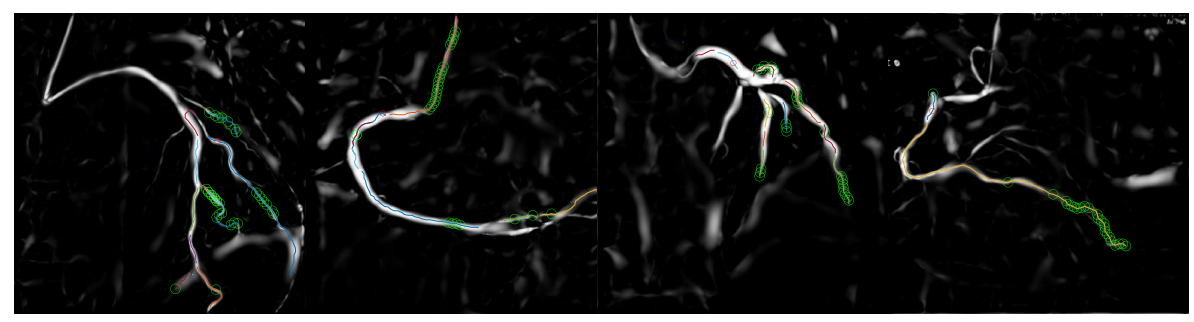

(a1)

$(\mathrm{a} 2)$

(a3)

$(\mathrm{a} 4)$

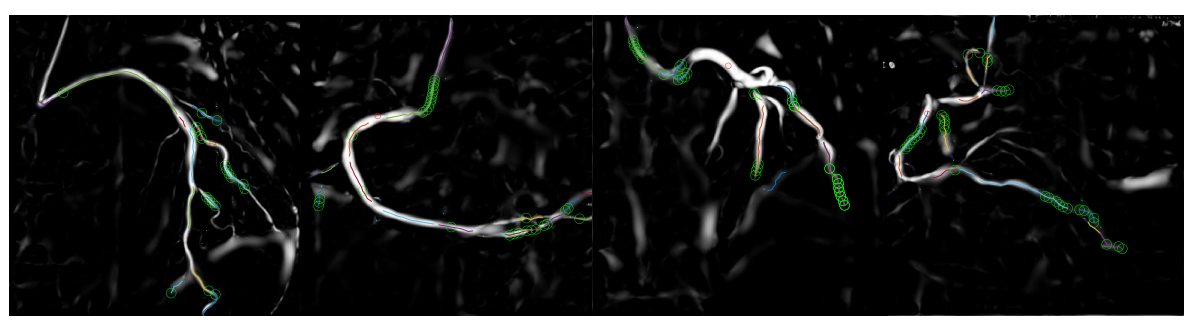

(b1)

(b2)

(b3)

(b4)

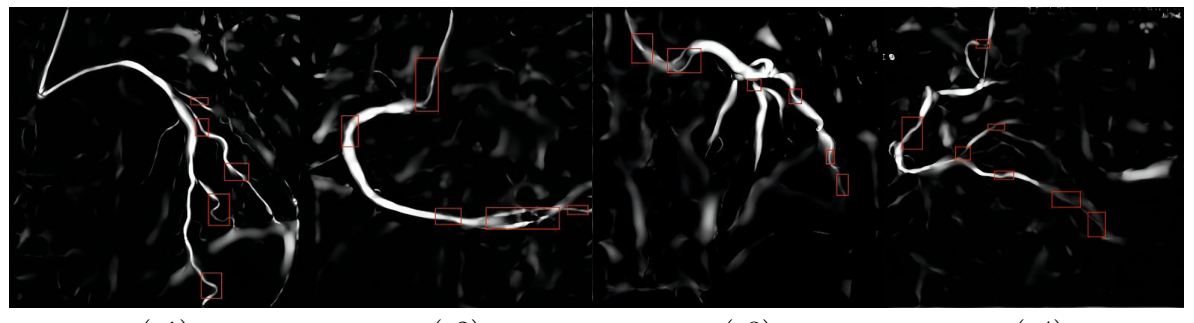

$(\mathrm{c} 1)$

$(\mathrm{c} 2)$

(c3)

(c4)

Fig. 7 Experimental results of automatic stenosis detection. (a) Xiao's method. (b) The proposed method. (c) Ground truth.

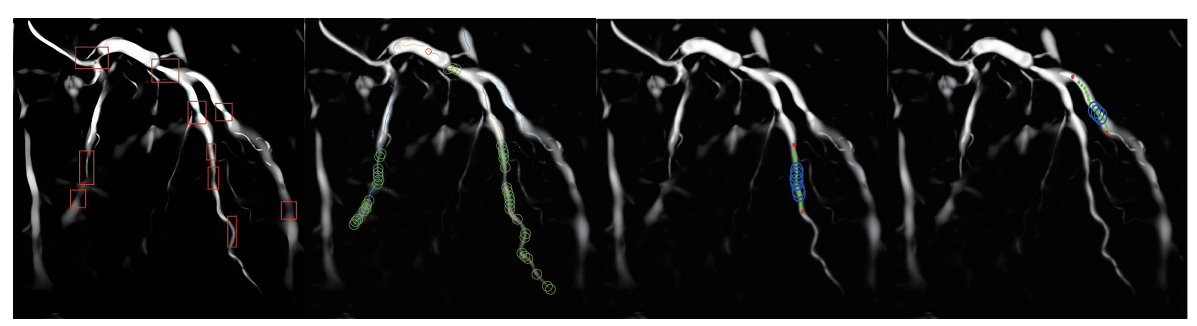

(a) (b)

(c1)

(c2)

Fig. 8 Results of more precise stenosis detection using the interactive method. (a) Ground truth. (b) Automatic method. (c) Interactive method.

In initial direction detection, search radius $r$ and angle $\Delta \theta$ are set to 5 pixels and $45^{\circ}$. In bifurcation detection, $\left(r_{1}, r_{2}\right)$ and $\Delta \theta^{\prime}$ are set to $(7,12)$ pixels and $135^{\circ}$ similarly. The values of thresholds $I_{0}, \tau_{P}, \tau_{1}, \tau_{2}, d, \tau_{B}, \tau_{3}, \lambda, \tau_{d}$ are set to $10,4,45^{\circ}, 30^{\circ}, 5,2,0.8,10000,5$ All the experiments are implemented on MATLAB software of version $2019 \mathrm{~b}$.

Preliminary results

To make a preliminary test on the effectiveness of our proposed solution, we selected three images with large differences in vessel structures for independent experiments and the results are shown in Fig.6. As can be seen from the figures, even though the vessel structures are different, the whole algorithm in this paper still has a good experimental effect on each image. Next, we will give more detailed and quantitative results of our methods. 


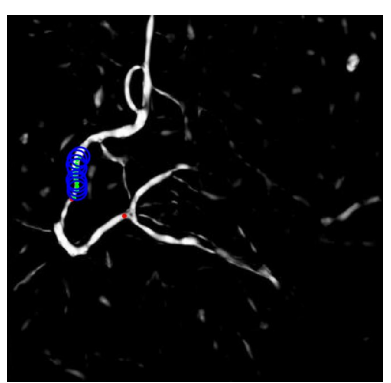

(a1)

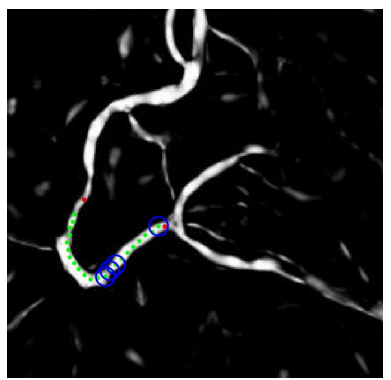

(b1)

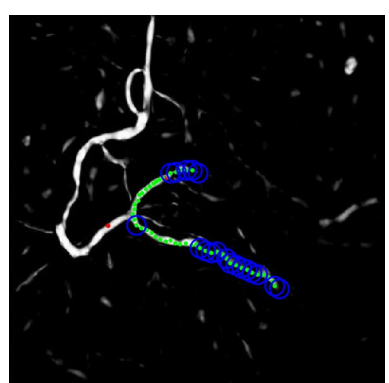

$(\mathrm{a} 2)$

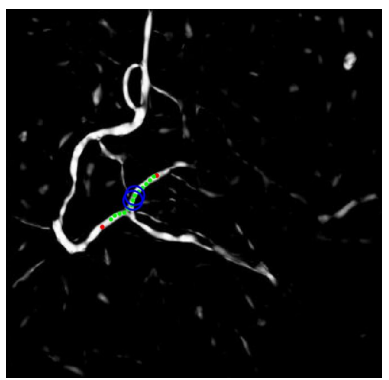

(b2)

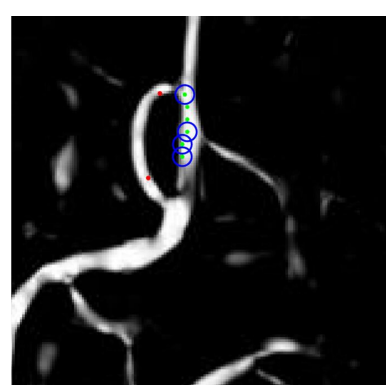

(a3)

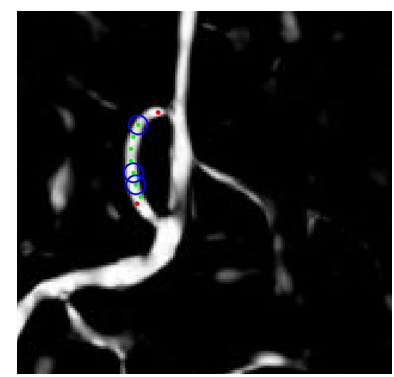

(b3)

Fig. 9 Comparison of the interactive stenosis detection before $((\mathrm{a}))$ and after $((\mathrm{b}))$ the introduction of the potential energy function

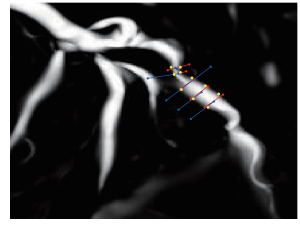

(a1)

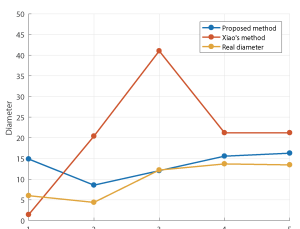

(b1)

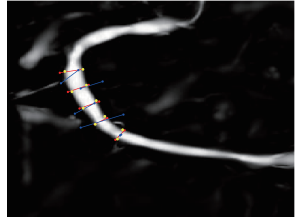

$(\mathrm{a} 2)$

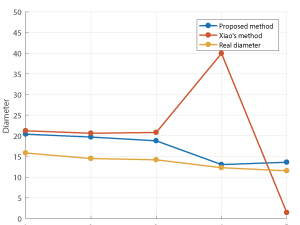

(b2)

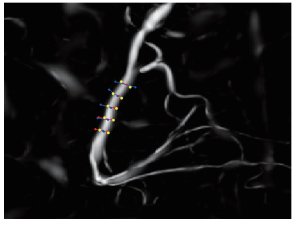

(a3)

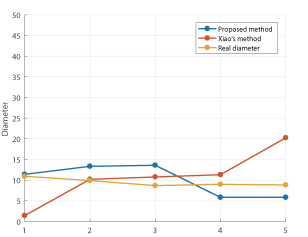

(b3)

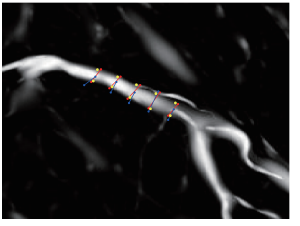

(a4)

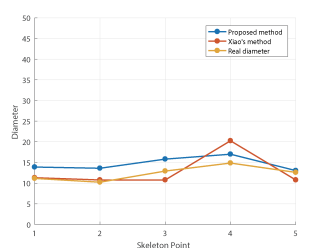

(b4)

Fig. 10 Comparison of the methods of diameter measurement. (a) Visual results (the red, blue, and yellow dots represent the intersection points obtained by the proposed method, the points obtained by Xiao's method, and the artificially marked points, respectively). (b) Line charts of the diameters (the abscissa is the serial number of the tracking points, the unit of diameters is a pixel).

\section{Study of automatic stenosis detection}

To further test the effectiveness of the proposed automatic method, we adopt Xiao's method [8], i.e., replace our tracking method and method of diameter measurement with hers in automatic stenosis detection, as a baseline. Fig. 7 shows the comparison of automatic stenosis detection between the proposed method and Xiao's method. We observe that our method can detect stenoses more accurately and make fewer mistakes. This is because we have a better tracking method and more precise diameter measurement, which will be discussed later.

For quantitative evaluations, we use three metrics, i.e., sensitivity(Sen), precision(Pre), and $F_{1}$-score, to measure the performance of the stenosis detection on the two methods. They are expressed as follows:

$$
\text { Sen }=\frac{T P}{T P+F N}
$$


Table 1 Comparison of automatic detection methods using three metrics

\begin{tabular}{llll}
\hline Algorithm & Sen & Pre & $F_{1}$ \\
\hline Xiao's Method $\left(\tau_{3}=0.8\right)$ & 0.466 & 0.697 & 0.592 \\
Proposed Method $\left(\tau_{3}=0.5\right)$ & 0.515 & 0.857 & 0.604 \\
Proposed Method $\left(\tau_{3}=0.8\right)$ & 0.757 & 0.821 & 0.788
\end{tabular}

$$
\begin{gathered}
\text { Pre }=\frac{T P}{T P+F P} \\
F_{1}=2 \times \frac{\text { Pre } \times \text { Sen }}{\text { Pre }+ \text { Sen }}
\end{gathered}
$$

where TP (true positive) is the number of stenoses that are detected correctly, FN (false negative) is the number of stenoses that are not detected, and FP (false positive) is the number of the points that are falsely detected as stenoses.

Table. 1 shows that the results of the proposed method are better than Xiao's method on all metrics. And the proposed $\operatorname{method}\left(\tau_{3}=0.8\right)$ improves the sensitivity while only sacrificing a little precision compared to $\left(\tau_{3}=0.5\right)$. As we focus more on detecting all the stenoses, it is accepted to make some incorrect predictions.

\section{Study of interactive stenosis detection}

To prove that the interactive method can do more precise detection than the automatic method, we use the interactive method to detect the stenoses that are not detected by the automatic method. The results are shown in Fig. 8. As can be seen, the interactive method can detect the labeled stenoses that are not detected by the automatic method.

The comparison of the detection results before and after the algorithm improvement, i.e., the introduction of the potential energy function, are shown in Fig. 9. By comparison, we can see that the improved algorithm has good robustness for arc structure, bifurcation structure, and even ring structure with large curvature.

To further evaluate the method of vessel diameter measurement, we compared the proposed method with Xiao's method. The artificially marked intersection points are ground truth represented by the yellow dots shown in Fig. 10. In Fig. 10 it can be observed that the points obtained by the proposed method are much closer to the marked ones, and so are the diameters.

To quantify the accuracy of the diameter estimation, we calculated the relative error $(R E)$, which is given by:

$$
R E=\frac{\left|R_{e}(P)-R_{r}(P)\right|}{R_{r}(P)}
$$

where $R_{e}(P)$ and $R_{r}(P)$ are the estimated and real vessel diameter at point $P$. The smaller values of $E R$ indicate better measures with lower estimation errors. Experiments were carried out on twenty vessel segments randomly selected from the dataset. Table. 2 gives the quantitative results, which contain the maximum, minimum, mean, and standard deviation (std) of $R E$. We can see that the proposed method achieved lower ER values than Xiao's method on almost every vessel segment, consistent with the visual results shown in Fig. 10 .

Overall, the results show that the proposed methods can handle more complex structures and have higher accuracy and stonger robustness than the former methods.

\section{Conclusions}

Detection and quantitative analysis of stenoses are of great significance to assist clinical diagnosis of CAD. In this paper, two new stenosis detection methods were proposed. The automatic stenosis detection method can automatically extract the coronary artery tree, calculate the diameters and stenotic degrees, and mark the stenoses. With the interactive stenosis detection method, only the starting point and the ending point 
Table 2 Relative error comparison of diameter measurement methods.

\begin{tabular}{ccccccccc}
\hline \multirow{2}{*}{ Vessels } & \multicolumn{7}{c}{ Proposed Method } & \multicolumn{3}{c}{ Xiao's Method } \\
\cline { 2 - 9 } V1 & 0.1549 & 0.8973 & 0.5209 & 0.2975 & 0.6672 & 3.0833 & 1.6888 & 1.0290 \\
V2 & 0.1264 & 0.6425 & 0.2720 & 0.2477 & 0.3399 & 3.2212 & 1.4208 & 1.2498 \\
V3 & 0.3021 & 0.7053 & 0.5607 & 0.1446 & 0.4446 & 3.0981 & 1.3213 & 0.9948 \\
V4 & 0.6921 & 0.7715 & 0.7217 & 0.0353 & 0.3063 & 0.8792 & 0.6210 & 0.2401 \\
V5 & 0.4498 & 0.5608 & 0.5176 & 0.0493 & 0.7539 & 4.5036 & 2.0560 & 1.7592 \\
V6 & 0.0639 & 0.1347 & 0.1082 & 0.0339 & 0.8848 & 2.7491 & 1.7200 & 0.9756 \\
V7 & 0.6102 & 0.7695 & 0.6817 & 0.0791 & 0.7755 & 1.2286 & 0.9379 & 0.200 \\
V8 & 0.2112 & 0.6163 & 0.4706 & 0.1596 & 1.8589 & 8.8833 & 4.5699 & 2.9516 \\
V9 & 0.0894 & 0.7293 & 0.3589 & 0.2469 & 0.7707 & 0.9005 & 0.8330 & 0.0515 \\
V10 & 0.0472 & 0.6809 & 0.4393 & 0.2668 & 0.7968 & 2.4050 & 1.7085 & 0.8012 \\
V11 & 0.0559 & 0.3039 & 0.2197 & 0.1018 & 0.8043 & 2.2939 & 1.4188 & 0.7808 \\
V12 & 0.0006 & 0.1345 & 0.0542 & 0.0587 & 0.5475 & 2.4841 & 1.0875 & 0.7914 \\
V13 & 0.5295 & 0.7150 & 0.6488 & 0.0733 & 0.6296 & 1.6901 & 1.0550 & 0.3971 \\
V14 & 0.0939 & 0.2676 & 0.1764 & 0.0674 & 0.6328 & 0.8850 & 0.8059 & 0.1073 \\
V15 & 0.4422 & 0.7050 & 0.5906 & 0.1094 & 0.7904 & 2.7043 & 1.2029 & 0.8405 \\
V16 & 0.5225 & 0.6906 & 0.6222 & 0.0790 & 0.7693 & 0.8777 & 0.8299 & 0.0434 \\
V17 & 0.0139 & 2.2251 & 0.4812 & 0.8641 & 0.0313 & 1.3661 & 0.7532 & 0.5476 \\
V18 & 0.1841 & 0.7091 & 0.4239 & 0.1956 & 0.8519 & 2.9889 & 1.3315 & 0.9289 \\
V19 & 0.1112 & 0.6417 & 0.4568 & 0.1823 & 0.8236 & 5.7977 & 2.1899 & 2.0836 \\
V20 & 0.0197 & 0.1703 & 0.0864 & 0.0711 & 0.2219 & 1.6625 & 1.0041 & 0.6869 \\
\hline Mean & 0.2360 & 0.6535 & 0.4206 & 0.1682 & 0.6851 & 2.6851 & 1.4278 & 0.8730 \\
\hline & & & & & & & &
\end{tabular}

need to be set artificially to get the target vessel segment and detect and analyze its stenoses. A large number of experiments showed that the two algorithms have strong robustness and high accuracy for vessels with different structures.

However, the methods in this paper also have some limitations, such as high dependence on the preprocessing and the vessel contour extraction algorithm, which will produce errors under some complex vessel structures. Therefore, in the following work, we will consider using more advanced methods like deep learning for preprocessing and vessel contour extraction to further improve the detection effect.

Funding Yaofang Liu, Xinyue Zhang, Wenlong Wan, Shaoyu Liu, Yingdi Liu, Hu Liu,and Xueying Zeng was supported by the National Natural Science Foundation of China [No.11771408], the Fundamental Research Funds for the Central Universities [No.201964006]. Qing Zhang was supported by the National Natural Science Foundation of China [No.81671703], the Key Research and Development Project of Shandong Province [No.2015GSF118026], the Qingdao Key Health Discipline Development Fund, and People's Livelihood Science and Technology Project of Qingdao [No.18-6-1-62-nsh]. Availability of data and materials Data is not available for this study.

\section{Declarations}

Conflict of interest Authors do not have any conflicts of interest.

Authors' contributions Yaofang Liu, Xinyue Zhang, and Wenlong Wan contributed equally.

Ethical approval This article does not contain any studies with human participants or animals performed by any of the authors.

Informed consent Not applicable.

Code availability Code is currently not made available.

\section{References}

1. Lars Husmann, Sebastian Leschka, Lotus Desbiolles, Tiziano Schepis, Oliver Gaemperli, Burkhardt Seifert, Philippe Cattin, Thomas Frauenfelder, Thomas G. Flohr, Borut Marincek, Philipp A. Kaufmann, and Hatem Alkadhi. Coronary Artery Motion and Cardiac Phases: Dependency on Heart Rate-Implications for CT Image Reconstruction. Radiology, 245(2):567-576, November 2007.

2. Gregory A Roth, Degu Abate, Kalkidan Hassen Abate, Solomon M Abay, Cristiana Abbafati, Nooshin Abbasi, Hedayat Abbastabar, Foad Abd-Allah, Jemal Abdela, Ahmed Abdelalim, et al. Global, regional, and national age-sex-specific mortality 
for 282 causes of death in 195 countries and territories, 1980-2017: a systematic analysis for the global burden of disease study 2017. The Lancet, 392(10159):17361788, 2018.

3. Salim S Virani, Alvaro Alonso, Emelia J Benjamin, Marcio S Bittencourt, Clifton W Callaway, April P Carson, Alanna M Chamberlain, Alexander R Chang, Susan Cheng, Francesca N Delling, et al. Heart disease and stroke statistics - 2020 update: a report from the american heart association. Circulation, 141(9):e139-e596, 2020.

4. Song Jiangping, Zheng Zhe, Wang Wei, Song Yunhu, Huang Jie, Wang Hongyue, Zhao Hong, and $\mathrm{Hu}$ Shengshou. Assessment of Coronary Artery Stenosis by Coronary Angiography: A Head-to-Head Comparison With Pathological Coronary Artery Anatomy. Circ Cardiovasc Interv, 6(3):262-268, June 2013.

5. Piotr Makowski, Thomas Sangild Sørensen, Søren Vorre Therkildsen, Andrzej Materka, Hans Stødkilde-Jørgensen, and Erik Morre Pedersen. Two-phase active contour method for semiautomatic segmentation of the heart and blood vessels from mri images for 3d visualization. Computerized Medical Imaging and Graphics, 26(1):9-17, 2002.

6. Juan F Carrillo, Marcela Hernández Hoyos, Eduardo E Dávila, and Maciej Orkisz. Recursive tracking of vascular tree axes in 3d medical images. International Journal of Computer Assisted Radiology and Surgery, 1(6):331-339, 2007.

7. Rashindra Manniesing, Max A Viergever, and Wiro J Niessen. Vessel axis tracking using topology constrained surface evolution. IEEE Transactions on Medical Imaging, 26(3):309-316, 2007.

8. Ruoxiu Xiao, Jian Yang, Mahima Goyal, Yue Liu, and Yongtian Wang. Automatic Vasculature Identification in Coronary Angiograms by Adaptive Geometrical Tracking. Computational and Mathematical Methods in Medicine, 2013:1-11, 2013.

9. Alejandro F. Frangi, Wiro J. Niessen, Koen L. Vincken, and Max A. Viergever. Multiscale vessel enhancement filtering. In William M. Wells, Alan Colchester, and Scott Delp, editors, Medical Image Computing and Computer-Assisted Intervention - MICCAI'98, Lecture Notes in Computer Science, pages 130-137, Berlin, Heidelberg, 1998. Springer.

10. Ivan Cruz-Aceves, Fernando Cervantes-Sanchez, and Maria Susana Avila-Garcia. A novel multiscale gaussian-matched filter using neural networks for the segmentation of x-ray coronary angiograms. Journal of healthcare engineering, 2018, 2018.

11. Kyungmin Jo, Jihoon Kweon, Young-Hak Kim, and Jaesoon Choi. Segmentation of the main vessel of the left anterior descending artery using selective feature mapping in coronary angiography. IEEE Access, 7:919-930, 2018.

12. Huihui Fang, Jianjun Zhu, Danni Ai, Yong Huang, Yurong Jiang, Hong Song, Yongtian Wang, and Jian Yang. Greedy soft matching for vascular tracking of coronary angiographic image sequences. IEEE Transactions on Circuits and Systems for Video Technology, 30(5):1466-1480, 2019.

13. Zhanchao Xian, Xiaoqing Wang, Shaodi Yan, Dahao Yang, Junyu Chen, and Changnong Peng. Main coronary vessel segmentation using deep learning in smart medical. Mathematical Problems in Engineering, 2020, 2020.

14. Ebrahim Nasr-Esfahani, Nader Karimi, Mohammad H Jafari, S Mohamad R Soroushmehr, Shadrokh Samavi, BK Nallamothu, and Kayvan Najarian. Segmentation of vessels in angiograms using convolutional neural networks. Biomedical Signal Processing and Control, 40:240-251, 2018.

15. Viacheslav V. Danilov, Kirill Yu. Klyshnikov, Olga M. Gerget, Anton G. Kutikhin, Vladimir I. Ganyukov, Alejandro F. Frangi, and Evgeny A. Ovcharenko. Realtime coronary artery stenosis detection based on modern neural networks. Sci Rep, 11(1):7582, December 2021.

16. Huazhu Fu, Yanwu Xu, Stephen Lin, Damon Wing Kee Wong, and Jiang Liu. Deepvessel: Retinal vessel segmentation via deep learning and conditional random field. In International conference on medical image computing and computer-assisted intervention, pages 132-139. Springer, 2016.

17. Zengqiang Yan, Xin Yang, and Kwang-Ting Cheng. A three-stage deep learning model for accurate retinal vessel segmentation. IEEE journal of Biomedical and Health Informatics, 23(4):1427-1436, 2018.

18. Américo Oliveira, Sergio Pereira, and Carlos A Silva. Retinal vessel segmentation based on fully convolutional neural networks. Expert Systems with Applications, 112:229-242, 2018 . 
19. Qiangguo Jin, Zhaopeng Meng, Tuan D Pham, Qi Chen, Leyi Wei, and Ran Su. Dunet: A deformable network for retinal vessel segmentation. Knowledge-Based Systems, 178:149-162, 2019.

20. Yicheng Wu, Yong Xia, Yang Song, Yanning Zhang, and Weidong Cai. Nfn+: A novel network followed network for retinal vessel segmentation. Neural Networks, 126:153-162, 2020.

21. Dongyi Wang, Ayman Haytham, Jessica Pottenburgh, Osamah Saeedi, and Yang Tao. Hard attention net for automatic retinal vessel segmentation. IEEE Journal of Biomedical and Health Informatics, 24(12):3384-3396, 2020.

22. Chunhui Chen, Joon Huang Chuah, Ali Raza, and Yizhou Wang. Retinal vessel segmentation using deep learning: A review. IEEE Access, 2021.

23. Chao Cong, Yoko Kato, Henrique Doria Vasconcellos, Joao Lima, and Bharath Venkatesh. Automated stenosis detection and classification in x-ray angiography using deep neural network. In 2019 IEEE International Conference on Bioinformatics and Biomedicine (BIBM), pages 1301-1308. IEEE, 2019.

24. Emmanuel Ovalle-Magallanes, Juan Gabriel Avina-Cervantes, Ivan Cruz-Aceves, and Jose Ruiz-Pinales. Transfer learning for stenosis detection in x-ray coronary angiography. Mathematics, 8(9):1510, 2020.

25. Leonid I. Rudin, Stanley Osher, and Emad Fatemi. Nonlinear total variation based noise removal algorithms. Physica D: Nonlinear Phenomena, 60(1-4):259 268, November 1992.

26. D. F. Malin. Unsharp masking. AAS Photo Bulletin, 16:10-13, January 1977.

27. S.M. Pizer, R.E. Johnston, J.P. Ericksen, B.C. Yankaskas, and K.E. Muller. Contrast-limited adaptive histogram equalization: speed and effectiveness. In [1990] Proceedings of the First Conference on Visualization in Biomedical Computing, pages 337-345, Atlanta, GA, USA, 1990. IEEE Comput. Soc. Press.

28. Mushtaq Ahmad Khan, Wen Chen, Asmat Ullah, and Zhuojia Fu. A mesh-free algorithm for ROF model. EURASIP Journal on Advances in Signal Processing, 2017(1):53, July 2017.

29. Guang Deng. A Generalized Unsharp Masking Algorithm. IEEE Transactions on Image Processing, 20(5):1249-1261, May 2011. Conference Name: IEEE Transactions on Image Processing.

30. Agung W. Setiawan, Tati R. Mengko, Oerip S. Santoso, and Andriyan B. Suksmono. Color retinal image enhancement using CLAHE. In International Conference on ICT for Smart Society, pages 1-3, June 2013.

31. T.F. Chan and L.A. Vese. Active contours without edges. IEEE Trans. on Image Process., 10(2):266-277, February 2001.

32. Yun Tian, Qingli Chen, Wei Wang, Yu Peng, Qingjun Wang, Fuqing Duan, Zhongke $\mathrm{Wu}$, and Mingquan Zhou. A Vessel Active Contour Model for Vascular Segmentation. BioMed Research International, 2014:106490, 2014. Publisher: Hindawi Publishing Corporation.

33. Anne M Neubauer, Joel A Garcia, John C Messenger, Eberhard Hansis, Michael S Kim, Andrew JP Klein, Gert AF Schoonenberg, Michael Grass, and John D Carroll. Clinical feasibility of a fully automated $3 \mathrm{~d}$ reconstruction of rotational coronary $\mathrm{x}-$ ray angiograms. Circulation: Cardiovascular Interventions, 3(1):71-79, 2010. 